\title{
Concordance of FDG PET/CT metabolic tumour volume versus DW-MRI functional tumour volume with T2-weighted anatomical tumour volume in cervical cancer
}

Alta Y. T. Lai ${ }^{1}$, Jose A. U. Perucho ${ }^{2}$, Xiaopei Xu², Edward S. Hui ${ }^{2}$ and Elaine Y. P. Lee ${ }^{2^{*}}$

\begin{abstract}
Background: ${ }^{18} \mathrm{~F}$-fluoro-deoxyglucose positron emission tomography with computed tomography (FDG PET/CT) has been employed to define radiotherapy targets using a threshold based on the standardised uptake value (SUV), and has been described for use in cervical cancer. The aim of this study was to evaluate the concordance between the metabolic tumour volume (MTV) measured on FDG PET/CT and the anatomical tumour volume (ATV) measured on T2-weighted magnetic resonance imaging (T2W-MRI); and compared with the functional tumour volume (FTV) measured on diffusion-weighted MRI (DW-MRI) in cervical cancer, taking the T2W-ATV as gold standard.
\end{abstract}

Methods: Consecutive newly diagnosed cervical cancer patients who underwent FDG PET/CT and DW-MRI were retrospectively reviewed from June 2013 to July 2017.

Volumes of interest was inserted to the focal hypermetabolic activity corresponding to the cervical tumour on FDG PET/CT with automated tumour contouring and manual adjustment, based on SUV $20 \%-80 \%$ thresholds of the maximum SUV (SUVmax) to define the MTV $20-80$, with intervals of $5 \%$.

Tumour areas were manually delineated on T2W-MRI and multiplied by slice thickness to calculate the ATV.

FTV were derived by manually delineating tumour area on ADC map, multiplied by the slice thickness to determine the $\mathrm{FTV}_{\text {(manual). }}$. Diffusion restricted areas was extracted from b0 and ADC map using K-means clustering to determine the FTV (semi-automated).

The ATVs, FTVs and the MTVs at different thresholds were compared using the mean and correlated using Pearson's product-moment correlation.

Results: Twenty-nine patients were evaluated (median age 52 years). Paired difference of mean between ATV and MTV was the closest and not statistically significant at $\operatorname{MTV}_{30}\left(-2.9 \mathrm{~cm}^{3},-5.2 \%, p=0.301\right)$. This was less than the differences between ATV and FTV (semi-automated) $\left(25.0 \mathrm{~cm}^{3}, 45.1 \%, p<0.001\right)$ and $\mathrm{FTV}_{\text {(manual) }}\left(11.2 \mathrm{~cm}^{3}, 20.1 \%, p=0.001\right)$. The correlation of $\mathrm{MTV}_{30}$ with ATV was excellent $(r=0.968, p<0.001)$ and better than that of the FTVs.

(Continued on next page)

\footnotetext{
* Correspondence: eyplee77@hku.hk

${ }^{2}$ Department of Diagnostic Radiology, Queen Mary Hospital, Li Ka Shing

Faculty of Medicine, The University of Hong Kong, Room 406, Block K, 102

Pokfulam Road, High West, Hong Kong Special Administrative Region, China

Full list of author information is available at the end of the article
} 
(Continued from previous page)

Conclusions: Our study demonstrated that $\mathrm{MTV}_{30}$ was the only parameter investigated with no statistically significant difference with ATV, had the least absolute difference from ATV, and showed excellent positive correlation with ATV, suggesting its superiority as a functional imaging modality when compared with DW-MRI and supporting its use as a surrogate for ATV for radiotherapy tumour contouring.

Keywords: Uterine cervical neoplasms, Positron-emission tomography, Fluorodeoxyglucose F18, Radiation oncology, Image-guided radiotherapy, Intensity-modulated radiotherapy

\section{Background}

Precise determination of cervical tumour boundary is important in radiotherapy to deliver the highest possible radiation dose to cancerous tissues while minimizing that to surrounding healthy tissues.

Given its superior soft tissue contrast, MRI is the modality of choice for the anatomical delineation of tumour outline and local tumour extent, especially in determining whether parametrial invasion is present to differentiate early from advanced stage disease. Despite excellent spatial resolution, delineation of tumour extent can be limited using conventional T2-weighted (T2W) sequences in certain scenarios, e.g. isointense tumours and diffusely infiltrative lesions, in assessing response of tumours to therapy and, in particular, in differentiating residual or recurrent disease from post-treatment fibrosis due to the overlap of morphological appearances [1].

The clinical utilisation of functional imaging in gynaecological malignancy is evolving [2-4]. In the era of more sophisticated treatment options such as image-guided adaptive radiotherapy, functional imaging techniques such as diffusion-weighted MRI (DW-MRI) and ${ }^{18} \mathrm{~F}$-fluorodeoxyglucose positron emission tomography integrated with computed tomography (FDG PET/CT) have been demonstrated to provide information for more precise definition of radiation target [5-7]. DW-MRI allows characterization of biological tissues based on their water diffusion property that changes with the integrity of cellular membranes and tissue cellularity [8]. Quantitative assessment can be derived from the apparent coefficient diffusion (ADC) maps obtained from DW-MRI [9]. For instance, ADC has been used to differentiate between normal and cancerous cervical tissue, and the latter was found to correlate negatively with tumour cellular density and grading $[10,11]$. Additional DW-MRI has been demonstrated in the literature to outperform $\mathrm{T} 2 \mathrm{~W}$ imaging alone in depicting local recurrence and differentiating it from post-treatment changes such as fibrosis [12-14]. The use of ADC for measuring target volumes with different tissue characteristics for dose prescription in image-guided adaptive brachytherapy [15] and various segmentation methods with DW-MRI [16] have also been studied. However, further investigation in its clinical application to radiotherapy treatment planning is warranted.
FDG PET/CT utilises FDG, a glucose analogue, to provide valuable metabolic information based on the increased glucose uptake and glycolysis of cancer cells, and can depict metabolic abnormalities before morphological alterations occur [17]. FDG PET/CT has been employed to define radiotherapy targets using a threshold based on the standardised uptake value (SUV) for over a decade [18], and that for cervical cancer has been recently demonstrated [19]. Modification of radiation treatment volumes to FDGavid lymph nodes and primary tumour can facilitate the accurate definition of tissues with metabolically active disease and the avoidance of normal tissue; hence allowing dose boosts to FDG-avid tumour volumes and lower doses to the bone marrow, urinary bladder and rectum $[20,21]$.

Despite promising results of using functional imaging to delineate radiation target in cervical cancer, the segmentation methods and thresholds used are highly variable in the literature. The aim of this study is to evaluate the concordance between the metabolic tumour volume (MTV) measured on FDG PET/CT and the anatomical tumour volume (ATV) measured on T2W imaging; and compared with the functional tumour volume (FTV) measured on DW-MRI in cervical cancer, using the T2W ATV as gold standard [22].

\section{Methods \\ Patient selection}

The retrospective study was reviewed by local institutional review board and informed consent was waived. We reviewed the local database and all consecutive patients with newly diagnosed cervical cancers who underwent both FDG PET/CT and MRI as pre-treatment imaging from June 2013 to July 2017 were included. Cases with incomplete inclusion of the tumour on MRI were excluded. The median time difference between the two examinations was 4 days (range 0 to 32).

\section{FDG PET/CT}

\section{Patient preparation and image acquisition}

Whole-body FDG PET/CT (coverage from the skull base to the upper one third of the thighs) was performed on a combined PET/CT scanner (Discovery VCT, 64 multislice spiral CT; GE Healthcare Bio-Sciences Corp.), using a standardised protocol. After $6 \mathrm{~h}$ of fasting, 222-370 MBq 
(4.8 MBq/kg) of weight-adjusted FDG was administered intravenously. Following a 60-min uptake time, whole-body emission PET was obtained with 6 bed positions of 2 min and $30 \mathrm{~s}$ acquisition time in each bed position. PET was attenuated with CT data and reconstructed with an orderedsubset expectation maximization iterative reconstruction algorithm (14 subsets and 2 iterations) and subsequently fused with CT images for further analysis. The CT imaging parameters were as follows: $120 \mathrm{kVp}$; 200-400 mA; $0.5 \mathrm{~s}$ per CT rotation; pitch, $0.984: 1$; and $2.5-\mathrm{mm}$ intervals, with or without $60-100 \mathrm{~mL}(1.5 \mathrm{~mL} / \mathrm{kg})$ intravenous contrast medium.

\section{Metabolic tumour volume (MTV)}

Both SUV and volumetric analysis were performed using Advantage Volume Share on ADW 4.7 workstation (GE Healthcare, Chicago, Illinois, United States). Focal hypermetabolic activity in the uterine cervix corresponding to the cervical tumour was visually identified, where a 3D volume of interest (VOI) was inserted (Fig. 1). Automated tumour contouring with manual adjustment was performed to include the boundaries of the lesion in the axial, coronal, and sagittal planes, and to avoid the urinary bladder. SUV measurement was performed by normalization of the injected dose to lean body mass. Lean body mass was used for normalization instead of total body mass because it is less dependent on body habitus across populations [23]. Maximum SUV (SUVmax) was automatically generated. MTV was measured using an SUV-based automated contouring program. The voxels presenting SUV $\geq 20 \%$ to $80 \%$ thresholds of the SUVmax within the contouring margin were incorporated to define the metabolic tumour volumes $\left(\mathrm{MTV}_{20}\right.$ to $\left.\mathrm{MTV}_{80}\right)$, with intervals of $5 \%$.

\section{MRI}

\section{Patient preparation and image acquisition}

Patients were prepared for MRI after $6 \mathrm{~h}$ of fasting and $20 \mathrm{mg}$ hyoscine butylbromide (Buscopan, Boehringer
Ingelheim, Germany) was given intramuscularly at the start of each examination to reduce bowel peristalsis. All examinations were performed on a 3.0-T MRI system (Achieva 3.0 T TX, Philips Healthcare, Best, the Netherlands) using a dedicated 16-channel phased array torso coil.

The standard sequences included sagittal T2 W turbo spin-echo (TSE) and an oblique axial T2W TSE (perpendicular to the long axis of the cervix). Additional axial T2W TSE was acquired to ensure the same anatomical coverage and slice profile as the DW-MRI. Post-contrast 3D T1 W TSE was acquired after DW-MRI (Table 1).

DW-MRI was performed using single-shot spin-echo echo-planar imaging, immediately after the axial T2W TSE imaging. It was acquired in free breathing with background body signal suppression (presaturation inversion recovery fat suppression) and parallel imaging with sensitivity encoding [SENSE] factor of 2 (Table 1). Image acquisition with $13 b$-values $\left(0-1000 \mathrm{~s} / \mathrm{mm}^{2}\right)$ were performed in the axial plane covering 20 slices to include the entire cervical cancer, using motion-probing gradients in three orthogonal axes to generate the geometric averaged DW signal. The full inclusion of the entirety of the tumour on the DW-MRI images was confirmed visually for every case.

\section{Anatomical tumour volume (ATV)}

Tumour areas were manually delineated on T2W images in sagittal and oblique axial planes and multiplied by the slice thickness to calculate the sagittal and oblique axial tumour volumes. Two reviewers, EL (8-year experience in MRI with special interest in gynaecological oncology imaging) and AL (5-year experience in MRI), separately placed the ROIs on the T2W images in the sagittal and oblique axial planes, respectively. The volumes were averaged between the two reviewers to determine the ATV.

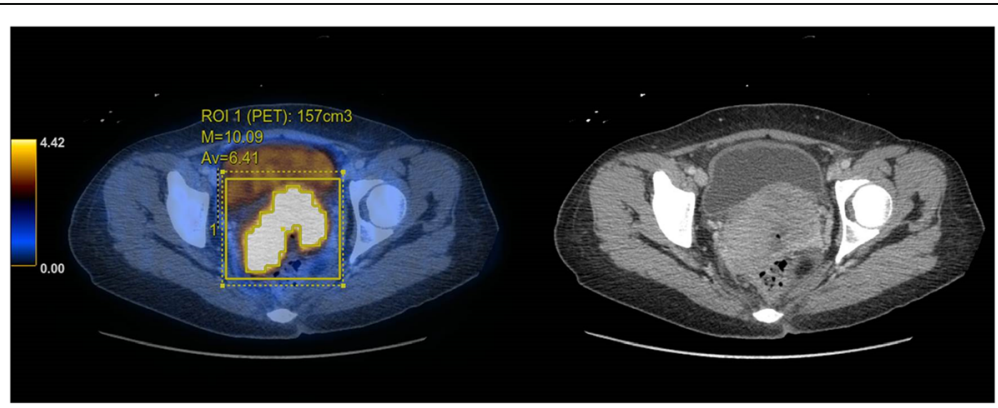

Fig. 1 MTV was calculated by the thresholding method on FDG PET/CT. Focal activity in the uterine cervix was identified. A VOI was inserted manually, carefully avoiding the urinary bladder. SUVmax was quantified by the software automatically. The tumour was outlined as the region encompassed by a given fixed percent intensity level relative to the maximum activity in the tumour. 20\% to 80\% thresholds of the SUVmax $\left(M_{T V} 20\right.$ to $\left.M T V_{80}\right)$ at intervals of $5 \%$ were used in this study. MTV: metabolic tumour volume; VOl: volume of interest; SUVmax: maximum standardized uptake value 
Table 1 Summary of MRI scan parameters

\begin{tabular}{|c|c|c|c|c|c|c|}
\hline Sequences & T2-Weighted TSE & T2-Weighted SPAIR & T2-Weighted TSE & T2-Weighted TSE & DWI & CE 3D T1-weighted TSE \\
\hline Plane & Sagittal & Coronal & Axial & Oblique Axial & Axial & $3 D$ \\
\hline TR/TE (ms) & $4000 / 80$ & $3500 / 80$ & $2800 / 100$ & $2800 / 100$ & $2000 / 54$ & $3 / 1.4$ \\
\hline Turbo factor & 30 & 21 & 12 & 14 & NA & NA \\
\hline Field of view (mm) & $240 \times 240$ & $230 \times 230$ & $402 \times 300$ & $220 \times 220$ & $406 \times 300$ & $370 \times 203$ \\
\hline Matrix size & $480 \times 298$ & $352 \times 300$ & $787 \times 600$ & $316 \times 311$ & $168 \times 124$ & $248 \times 134$ \\
\hline Slice thickness (mm) & 4 & 4 & 4 & 4 & 4 & 1.5 \\
\hline Intersection gap (mm) & 0 & 0 & 0 & 0 & 0 & 0 \\
\hline Bandwidth (Hz/pixel) & 230 & 186 & 169 & 162 & 15.3 & 724 \\
\hline Number of excitations & 2 & 1 & 1 & 1 & 2 & 1 \\
\hline
\end{tabular}

CE: contrast-enhanced, DWI: diffusion-weighted imaging; TR/TE: repetition time/echo; TSE: turbo spin echo

\section{Functional tumour volume (FTV)}

Averaged DW signal was used to generate the ADC maps using the Levenberg-Marquardt fitting algorithm under the mono-exponential model described by the function:

$$
\frac{S_{b}}{S_{0}}=\left[e^{-b \cdot A D C}\right]
$$

where $S_{b}$ represents the mean signal intensity with the diffusion gradient, $b, \mathrm{~S}_{0}$ is the mean signal intensity when $b=0 \mathrm{~s} / \mathrm{mm}^{2}$. VOIs were manually drawn by two reviewers, EL and AL, for each lesion. The first set of VOIs were strict manual delineations of the tumour by both reviewers and excluded the surrounding normal tissue based on the hypointense signal of the tumour on the ADC map with cross reference to the axial T2W images. FTV by the two reviewers was then calculated using these VOIs multiplied by slice thickness. The volumes were averaged between the two reviewers to determine the $\mathrm{FTV}_{\text {(manual) }}$. The second set of VOIs was drawn by the same two reviewers, EL and AL, to include all of the tumour and did not require exclusion of surrounding normal tissue. Volumetric k-means clustering was then used to automatically separate voxels in the tumour volume into three groups based on $S_{0}$ and ADC values. These groups were defined as: solid tumour mass with high cellularity having intermediate $\mathrm{ADC}$ and intermediate $\mathrm{S}_{0}$ intensities; normal tissue with low cellularity or cystic tissues having high $\mathrm{ADC}[5,24]$ and high $\mathrm{S}_{0}$ intensities; fat and fibrotic tissues having low ADC low $\mathrm{S}_{0}$ intensities. A study by Gong et al. [25] has shown that slice-by-slice K-means clustering, using both $\mathrm{S}_{0}$ images and ADC, is a promising method for reliable delineation of heterogeneous tumours in patients with metastatic gastrointestinal stromal tumours. FTV (semi-automated) was calculated by discarding the fat and fibrotic cluster and the normal tissue cluster, leaving the solid tumour mass cluster. Parametric map generation and semi-automatic functional volume segmentation were performed using in-house programs using MATLAB (The Mathworks Inc., Natick, MA, USA) (Fig. 2). The volumes were averaged between the two reviewers to determine the $\mathrm{FTV}_{\text {(semi-automated). }}$

\section{Statistical analysis}

The ATVs measured on T2W images, FTVs on DW-MRI and the MTVs at different thresholds on FDG PET/CT were compared. The ATVs, FTVs and MTVs were correlated using Pearson's product-moment correlation. R version 3.4.1

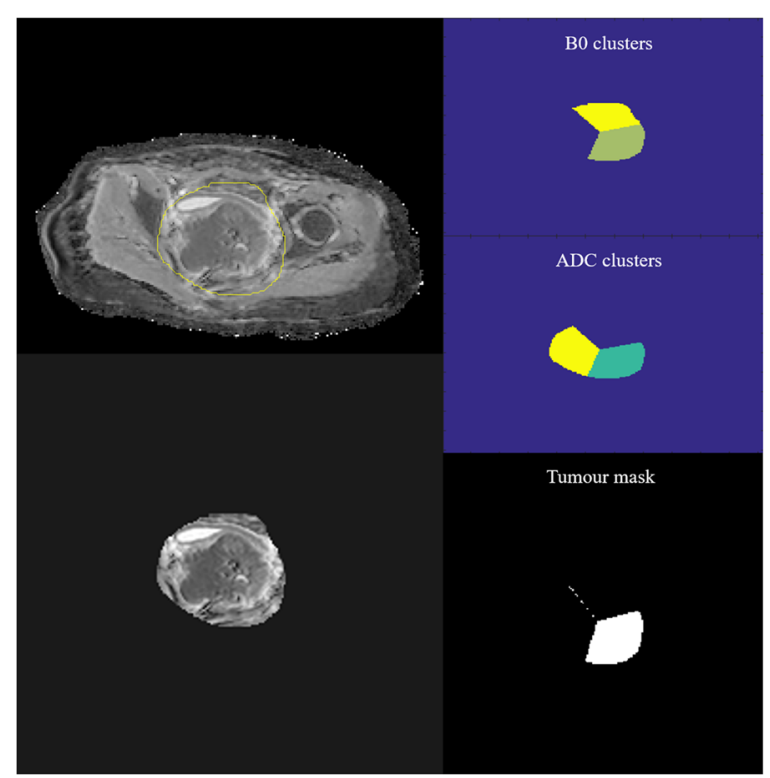

Fig. 2 A semi-automated method was used to extract diffusion restricted areas from corresponding 60 and ADC map. VOls were manually inserted to include the entire tumour. Voxels were automatically separated into 3 groups based on ADC values using a K-means clustering method: solid tumour mass with high cellularity having intermediate ADC, fat and fibrotic tissues having low ADC and normal tissue with low cellularity or cystic tissues having high ADC. FTV (semi-automated) was hence calculated by discarding the fat and fibrotic cluster and the normal tissue cluster, leaving the solid tumour mass cluster. ADC: apparent diffusion coefficient; FTV: functional tumour volume 
(R Foundation for Statistical Computing, Vienna, Austria) was used for statistical analysis. A two-tailed $p$-value $<0.05$ was considered statistically significant.

\section{Results}

\section{Demographics}

A total of 29 patients were evaluated with median age of 52 years (range 27-76 years). Further clinicopathological characteristics were tabulated in Table 2 .

\section{Quantitative measurements}

Mean SUVmax of the cervical tumours was 9.2, range 3.316.7. Mean ADC of the cervical tumours was $0.934+/-$ $0.120 \mathrm{~mm}^{2} / \mathrm{s}$. The mean ATVs measured in sagittal and oblique axial planes are 51.7 and $59.3 \mathrm{~cm}^{3}$ respectively.

\section{Paired differences of mean between (ATV and FTV) vs (ATV and MTV at different SUV thresholds)}

The paired difference of mean between ATV and $\mathrm{MTV}_{30}$, mathematically represented by mean ATV - mean $\mathrm{MTV}_{30}$, was $-2.9 \mathrm{~cm}^{3},-5.2 \%, p=0.301$. This difference was not statistically significant and was the closest to ATV compared with all other FTVs and MTVs measured at other SUVmax thresholds, including the differences between ATV and FTV $_{\text {(semi-automated) }}$ (mean ATV - mean FTV (semi-automated) $_{\text {(m) }}$ $\left.25.1 \mathrm{~cm}^{3}, 45.1 \%, p<0.001\right)$ and between ATV and $\mathrm{FTV}_{\text {(manual) }}$ (mean ATV - mean FTV $\mathrm{Fmanal}_{\text {(manu }}=11.2 \mathrm{~cm}^{3}$, $20.1 \%, p=0.001)$. The means of the ATV, FTV (semi-automated), $\mathrm{FTV}_{\text {(manual) }}$ and $\mathrm{MTV}_{20}$ to $\mathrm{MTV}_{80}$ (with intervals of $5 \%$ ) and the differences of their means with ATV are shown in Table 3.

\section{Correlation}

The correlations of $\mathrm{MTV}_{20-50}$ with ATV were excellent $(r>0.9, p<0.001)$, and those of $\mathrm{MTV}_{30-40}$ were better

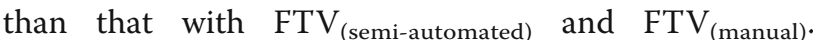
There was a gradual decline in correlation of MTVs

Table 2 Patient demographics including age, clinical tumour staging and histological types

\begin{tabular}{lll}
\hline Median age in years (range) & $\begin{array}{l}52(27-76) \\
\text { No of patients, } \\
n(\%)\end{array}$ \\
\hline FIGO stage & IB & $8(27.6 \%)$ \\
& IIA & $2(6.9 \%)$ \\
& IIB & $7(24.1 \%)$ \\
III & $10(34.5 \%)$ \\
Histology & Unstaged & $1(3.4 \%)$ \\
& Squamous cell carcinoma & $1(3.4 \%)$ \\
& Adenocarcinoma & $8(27.6 \%)$ \\
& Others & $5(17.2 \%)$ \\
\hline
\end{tabular}

Table 3 Means of the ATV, FTV (semi-automated), $F T V_{\text {(manual) }}$ and $\mathrm{MTV}_{20}$ to $\mathrm{MTV}_{80}$

\begin{tabular}{|c|c|c|c|c|}
\hline & Mean $\left(\mathrm{cm}^{3}\right)$ & $\begin{array}{l}\text { Paired difference with } \\
\text { mean ATV }\left(\mathrm{cm}^{3}\right)\end{array}$ & (\%) & $p$ value \\
\hline ATV & 55.5 & & & \\
\hline $\mathrm{FTV}_{\text {(manual) }}$ & 44.3 & 11.2 & $20.1 \%$ & 0.001 \\
\hline $\mathrm{FTV}_{\text {(semi-automated) }}$ & 30.5 & 25.1 & $45.1 \%$ & $<0.001$ \\
\hline PET MTV 20 & 83.3 & -27.8 & $-50.0 \%$ & $<0.001$ \\
\hline PET MTV 25 & 69.2 & -13.7 & $-24.7 \%$ & 0.001 \\
\hline PET MTV 30 & 58.4 & -2.9 & $-5.2 \%$ & 0.301 \\
\hline PET MTV 35 & 49.0 & 6.5 & $11.8 \%$ & 0.026 \\
\hline PET MTV 40 & 41.4 & 14.1 & $25.3 \%$ & 0.001 \\
\hline PET MTV 45 & 34.9 & 20.6 & $37.1 \%$ & $<0.001$ \\
\hline PET MTV 50 & 29.3 & 26.2 & $47.2 \%$ & $<0.001$ \\
\hline PET MTV 55 & 24.3 & 31.2 & $56.2 \%$ & $<0.001$ \\
\hline PET MTV 60 & 19.4 & 36.1 & $65.0 \%$ & $<0.001$ \\
\hline PET MTV 65 & 15.0 & 40.5 & $73.0 \%$ & $<0.001$ \\
\hline PET MTV & 11.0 & 44.5 & $80.2 \%$ & $<0.001$ \\
\hline PET MTV 75 & 7.3 & 48.2 & $86.9 \%$ & $<0.001$ \\
\hline PET MTV 80 & 4.4 & 51.1 & $92.1 \%$ & $<0.001$ \\
\hline
\end{tabular}

The differences of their means with ATV. The paired difference of mean between ATV and MTV 30 was not statistically significant and was the closest to ATV compared with all other FTVs and MTVs (BOLD)

with ATV as the percentage threshold of SUVmax increased (Table 4).

\section{Discussion}

Our study demonstrated that among all metabolic threshold levels and FTV (semi-automated) and FTV (manual), the $\mathrm{MTV}_{30}$ had the least absolute difference from ATV and was the only parameter investigated which did not show a statistically significant difference from ATV. In addition, $\mathrm{MTV}_{30}$ showed excellent positive correlation with ATV.

MRI is indispensable in the local disease assessment of cervical cancer. The ability of combined functional volume assessment and local disease extent using MRI alone could present as a promising imaging algorithm for patients with cervical cancer. However, the evidence to support this is limited in the current literature and most studies have used the manual segmentation method based on DW-MRI images [26, 27].

The choice of imaging modality used in tumour contouring or segmentation technique can result in varying derived tumour volume $[16,28,29]$. There is no consensus of the methodology of tumour segmentation using DW-MRI or ADC values. Clustering is a method, which groups similar data, and the k-means algorithm is often chosen for image segmentation and grouping voxels of same signal intensities and has been used for classification of functional imaging data [30]. This algorithm is 
Table 4 Correlation of MTVs at all SUVmax thresholds were significantly correlated with $\mathrm{T} 2 \mathrm{~W}$ anatomical volume and with DW-MRI functional volume $(p<0.05)$

\begin{tabular}{|c|c|c|}
\hline & $\begin{array}{l}\text { Pearson's product-moment } \\
\text { correlation (r) }\end{array}$ & $p$ value \\
\hline ATV & 1.000 & \\
\hline $\mathrm{FTV}_{\text {(manual) }}$ & 0.963 & $<0.001$ \\
\hline FTV (semi-automated) & 0.956 & $<0.001$ \\
\hline PET MTV 20 & 0.955 & $<0.001$ \\
\hline PET MTV 25 & 0.962 & $<0.001$ \\
\hline PET MTV 30 & 0.968 & $<0.001$ \\
\hline PET MTV 35 & 0.975 & $<0.001$ \\
\hline PET MTV 40 & 0.968 & $<0.001$ \\
\hline PET MTV 45 & 0.948 & $<0.001$ \\
\hline PET MTV 50 & 0.921 & $<0.001$ \\
\hline PET MTV 55 & 0.877 & $<0.001$ \\
\hline PET MTV 60 & 0.830 & $<0.001$ \\
\hline PET MTV 65 & 0.803 & $<0.001$ \\
\hline PET MTV 70 & 0.777 & $<0.001$ \\
\hline PET MTV 75 & 0.777 & $<0.001$ \\
\hline PET MTV 80 & 0.781 & $<0.001$ \\
\hline
\end{tabular}

Pearson correlation coefficient ( $r$ ) larger than 0.9 are highlighted in bold. ATV: anatomical tumour volume; FTV: functional tumour volume; MTV: metabolic tumour volume

simple and efficient and has been shown to be able to differentiate ADC values of benign and malignant pathologies [24]. A recent study has shown that $\mathrm{K}$-means clustering using both $\mathrm{S}_{0}$ and $\mathrm{ADC}$ is a promising method for reliable delineation of heterogeneous tumours in patients with metastatic gastrointestinal stromal tumours [25]. The relative signal intensity [31] and region growing [32] methods are alternative segmentation techniques which were described to have limitations related to their dependence on $b$-value and acquisition method for DW-MRI images, and sensitivity to signal-to-noise ratio [16].

FDG PET/CT has the advantage of identifying the metabolic activity and providing information on tumour biology. It is increasingly recognized as a useful tool for directing RT planning during intensity-modulated radiation therapy, volumetric-modulated arc treatment [20] and image-guided brachytherapy [33], thereby allowing targeted dose escalation to target tissues with high metabolic activity, and reducing dose to surrounding tissues [34]. The utility of FDG PET/CT has been shown to lead to less gastrointestinal toxicity in patients with gynaecological malignancies [35].

Various tumour segmentation techniques using FDG PET/CT exist: manual contouring, which consists of visual assessment for determining tumour outline; thresholding, which uses a minimum SUV value to identify target; and gradient edge detection, in which tumour delineation is based on the changes in signal across a given area [36]. As SUV thresholding has been the focus in initial investigative approaches and is the most commonly employed method of FDG/PET-based tumour volume segmentation [18], it was the segmentation technique of choice in this study.

Volume concordance between FDG PET/CT, and T2W and DW-MRI imaging in cervical cancer has been previously observed [26, 27, 37], and tumour sub-volumes with increased metabolic activity on FDG PET/CT was found to have greater cell density by DW-MRI [38]. Zhang et al. suggested that PET-measured gross tumour volume using an SUVmax threshold method may increase the accuracy in target volume delineation when performed on a sequential FDG PET/MRI platform [37]. SUV-based primary squamous histology cervical tumour volume estimation at $30 \%$ to $35 \%$ of SUVmax values correlated significantly with volume on MRI [27]. In a hybrid FDG PET/MRI study, volume measurement using $35 \%$ or $40 \%$ thresholds of the SUVmax has been found to display a strong concordance with the tumour volumes measured on T2W and DWMRI in cervical cancer [27, 37].

In our study, the mean of differences between ATV and MTV was the smallest with $\mathrm{MTV}_{30}$, concordant with previous literature [27]. Although DW-MRI also gives information on tumour cellularity and the semiautomated method may potentially reduce processing time and inter-observer variability, our study suggested that the FTVs, regardless of the segmentation methods, borne larger differences from the ATV than $\mathrm{MTV}_{30}$ did. Moreover, contouring based on FDG PET/CT can be performed in a semi-automated fashion and this feature is readily available on standard workstation, which is easy to use, providing the merit of reducing the time required for processing, and potentially also improving interobserver agreement, as shown previously by studies on tumour delineation for rectal and lung cancers [39-41].

Furthermore, $\mathrm{MTV}_{30}$, having the least absolute difference from ATV, being the only parameter investigated with no statistically significant difference from ATV, and having an excellent positive correlation with ATV supported its use as a surrogate for ATV for radiotherapy tumour contouring and dose escalation; with the benefit of having metabolic information available for characterizing the biological features of the tumour and optimizing the use of individualized, conformal and biologically effective radiation therapy.

\section{Conclusion}

In conclusion, MTV delineation on FDG PET/CT appears promising and superior as a functional imaging modality when compared with DW-MRI in tumour contouring with $\mathrm{MTV}_{30}$ being the best correlate to ATV. 


\section{Abbreviations}

ADC: apparent diffusion coefficient; ATV: anatomical tumour volume; CE: contrast-enhanced; DW-MRI: diffusion weighted magnetic resonance imaging; FDG PET/CT: ${ }^{18} \mathrm{~F}$-fluoro-deoxyglucose positron emission tomography with computed tomography; FTV: functional tumour volume; MRI: magnetic resonance imaging; MTV: metabolic tumour volume; PET: positron emission tomography; SUV: standardised uptake value; SUVmax: maximum standardised uptake value; T2W: T2-weighted; TR/ TE: repetition time/echo; TSE: turbo spin echo; VOI: 3D volume of interest

\section{Acknowledgements}

Not applicable.

\section{Funding}

This research was partially supported by the General Research Fund (GRF, No. 17119916) of the Research Grants Council (RGC), Hong Kong. The RGC invites applications for research grants, approves awards through the GRF from funds made available by the Hong Kong government, and monitors the implementation of successful grants. The RGC does not influence the design of the study, collection, analysis, interpretation of the data, and in writing the manuscript.

\section{Availability of data and materials}

The datasets used and/or analysed during the current study are available from the corresponding author on reasonable request. These data are primary data obtained by one of the authors.

\section{Authors' contributions}

$\mathrm{AL}$, the first author, segmented and calculated the tumour volumes on DW-MRI segmented and calculated the tumour volumes on PET/CT and T2W-MRI; analysed the data; and was a major contributor in writing the manuscript. JP provided skills in automated tumour volume segmentation and calculation on MRI; segmented and calculated the tumour volumes on DW-MRI; analysed the data; and was a major contributor in writing the manuscript. XX and $\mathrm{EH}$ provided skills in automated tumour volume segmentation and calculation on MRl; and contributed in editing the manuscript. EL, the senior author, obtained ethics approval for the study; retrieved the patient list; segmented and calculated the tumour volumes on DW-MRl; segmented and calculated tumour volumes on PET/CT and T2W-MRI; and was a major contributor in writing the manuscript. All authors read and approved the final manuscript.

\section{Ethics approval and consent to participate}

Ethics approval was obtained from Institutional Review Board of the University of Hong Kong/Hospital Authority Hong Kong West Cluster (HKU/ HA HKWC IRB), IRB Reference Number UW 16-521. The study is a retrospective study involving human data that had already been collected and did not require additional recruitment of human subjects, waiving the need for informed consent.

\section{Consent for publication}

Not applicable.

\section{Competing interests}

The authors declare that they have no competing interests.

\section{Publisher's Note}

Springer Nature remains neutral with regard to jurisdictional claims in published maps and institutional affiliations.

\section{Author details}

'Department of Radiology, Pamela Youde Nethersole Eastern Hospital, Chai Wan, Hong Kong Special Administrative Region, China. ${ }^{2}$ Department of Diagnostic Radiology, Queen Mary Hospital, Li Ka Shing Faculty of Medicine, The University of Hong Kong, Room 406, Block K, 102 Pokfulam Road, High West, Hong Kong Special Administrative Region, China.
Received: 16 March 2017 Accepted: 20 November 2017

Published online: 06 December 2017

\section{References}

1. Nougaret S, Tirumani SH, Addley H, Pandey H, Sala E, Reinhold C. Pearls and pitfalls in MRI of gynecologic malignancy with diffusion-weighted technique. Am J Roentgenol. 2013;200(2):261-76.

2. Lee EYP, Khong P-L, Tse KY, Chan KKL, Chu MMY, Ngan HYS. Differentiation of aggressive and indolent subtypes of uterine sarcoma using maximum standardized uptake value. Nucl Med Commun. 2013;34(12):1185.

3. Lee EYP, Perucho JAU, Vardhanabhuti V, He J, Siu SWK, Ngu SF, Mayr NA, Yuh WT, Chan Q, Khong PL. Intravoxel incoherent motion MRI assessment of chemoradiation-induced pelvic bone marrow changes in cervical cancer and correlation with hematological toxicity. J Magn Reson Imaging. 2017.

4. Yu X, Lee EYP, Lai V, Chan Q. Correlation between tissue metabolism and cellularity assessed by standardized uptake value and apparent diffusion coefficient in peritoneal metastasis. J Magn Reson Imaging. 2014;40(1):99-105.

5. Chen J, Zhang Y, Liang B, Yang Z. The utility of diffusion-weighted MR imaging in cervical cancer. Eur J Radiol. 2010;74(3):e101-6.

6. Lin LL, Mutic S, Low DA, LaForest R, Vicic M, Zoberi I, Miller TR, Grigsby PW. Adaptive brachytherapy treatment planning for cervical cancer using FDGPET. Int J Radiat Oncol Biol Phys. 2007;67(1):91-6.

7. Malyapa RS, Mutic S, Low DA, Zoberi I, Bosch WR, Laforest R, Miller TR, Grigsby PW. Physiologic FDG-PET three-dimensional brachytherapy treatment planning for cervical cancer. Int J Radiat Oncol Biol Phys. 2002; 54(4):1140-6.

8. Koh D-M, Collins DJ, Diffusion-weighted MRI. In the body: applications and challenges in oncology. Am J Roentgenol. 2007;188(6):1622-35.

9. Punwani S. Diffusion weighted imaging of female pelvic cancers: concepts and clinical applications. Eur J Radiol. 2011;78(1):21-9.

10. Lee EY, Yu X, Chu MM, Ngan HY, Siu SW, Soong IS, Chan Q, Khong PL. Perfusion and diffusion characteristics of cervical cancer based on intraxovel incoherent motion MR imaging-a pilot study. Eur Radiol. 2014:24(7):1506-13.

11. Liu Y, Bai R, Sun H, Liu H, Zhao X, Li Y. Diffusion-weighted imaging in predicting and monitoring the response of uterine cervical cancer to combined chemoradiation. Clin Radiol. 2009;64(11):1067-74.

12. Bourgioti $C$, Chatoupis K, Moulopoulos LA. Current imaging strategies for the evaluation of uterine cervical cancer. World J Radiol. 2016;8(4):342.

13. Lucas R, Dias JL, Cunha TM. Added value of diffusion-weighted MRI in detection of cervical cancer recurrence: comparison with morphologic and dynamic contrast-enhanced MRI sequences. Diagn Interv Radiol. 2015;21(5):368.

14. Nishie A, Stolpen AH, Obuchi M, Kuehn DM, Dagit A, Andresen K. Evaluation of locally recurrent pelvic malignancy: performance of T2-and diffusionweighted MRI with image fusion. J Magn Reson Imaging. 2008;28(3):705-13.

15. Haack S, Pedersen EM, Jespersen SN, Kallehauge JF, Lindegaard JC, Tanderup K. Apparent diffusion coefficients in GEC ESTRO target volumes for image guided adaptive brachytherapy of locally advanced cervical cancer. Acta Oncol. 2010:49(7):978-83.

16. Haack S, Tanderup K, Kallehauge JF, Mohamed SMI, Lindegaard JC, Pedersen EM, Jespersen SN. Diffusion-weighted magnetic resonance imaging during radiotherapy of locally advanced cervical cancer-treatment response assessment using different segmentation methods. Acta Oncol. 2015:54(9):1535-42.

17. Almuhaideb A, Papathanasiou N, Bomanji J. 18 F-FDG PET/CT imaging in oncology. Ann Saudi Med. 2011;31(1):3.

18. Black QC, Grills IS, Kestin LL, C-YO W, Wong JW, Martinez AA, Yan D. Defining a radiotherapy target with positron emission tomography. Int Radiat Oncol Biol Phys. 2004;60(4):1272-82.

19. Erlich F, Camisão C, Nogueira-Rodriques A, Altino S, Ferreira C, Mamede M. 18 F-FDG-PET-based tumor delineation in cervical cancer: threshold contouring and lesion volumes. Revista Española de Medicina Nuclear e Imagen Molecular (English Edition). 2013;32(3):162-6.

20. Arnesen MR, Rekstad BL, Stokke C, Bruheim K, Løndalen AM, Hellebust TP, Malinen E, Short-course PET. Based simultaneous integrated boost for locally advanced cervical cancer. Radiat Oncol. 2016;11(1):39.

21. Magné N, Chargari C, Vicenzi L, Gillion N, Messai T, Magné J, Bonardel G, Haie-Meder C. New trends in the evaluation and treatment of cervix cancer: the role of FDG-PET. Cancer Treat Rev. 2008;34(8):671-81.

22. Dimopoulos JC, Petrow P, Tanderup K, Petric P, Berger D, Kirisits C, Pedersen EM, Van Limbergen E, Haie-Meder C, Pötter R. Recommendations from Gynaecological (GYN) GEC-ESTRO working group (IV): basic principles and 
parameters for MR imaging within the frame of image based adaptive cervix cancer brachytherapy. Radiother Oncol. 2012;103(1):113-22.

23. Wahl RL, Jacene H, Kasamon Y, Lodge MA. From RECIST to PERCIST: evolving considerations for PET response criteria in solid tumors. J Nucl Med. 2009;50(Suppl 1):122S-50S.

24. Srinivasan A, Galbán C, Johnson T, Chenevert T, Ross B, Mukherji S. Utility of the k-means clustering algorithm in differentiating apparent diffusion coefficient values of benign and malignant neck pathologies. Am J Neuroradiol. 2010;31(4):736-40.

25. Gong N-J, Wong C-S, Chu Y-C, Guo H, Huang B, Chan Q. Increasing the accuracy of volume and ADC delineation for heterogeneous tumor on diffusion-weighted MRI: correlation with PET/CT. Int J Radiat Oncol Biol Phys. 2013;87(2):407-13.

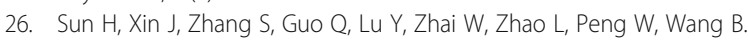
Anatomical and functional volume concordance between FDG PET, and T2 and diffusion-weighted MRI for cervical cancer: a hybrid PET/MR study. Eur J Nucl Med Mol Imaging. 2014;41(5):898-905.

27. Upasani MN, Mahantshetty UM, Rangarajan V, Purandare N, Merchant N, Thakur M, Engineer R, Chopra S, Shrivastava SK. 18-fluoro-deoxy-glucose positron emission tomography with computed tomography-based gross tumor volume estimation and validation with magnetic resonance imaging for locally advanced cervical cancers. Int J Gynecol Cancer. 2012;22(6):1031-6.

28. Ford EC, Kinahan PE, Hanlon L, Alessio A, Rajendran J, Schwartz DL, Phillips M. Tumor delineation using PET in head and neck cancers: threshold contouring and lesion volumes. Med Phys. 2006;33(11):4280-8.

29. Nestle U, Kremp S, Schaefer-Schuler A, Sebastian-Welsch C, Hellwig D, Rübe C, Kirsch C-M. Comparison of different methods for delineation of 18 F-FDG PET-positive tissue for target volume definition in radiotherapy of patients with non-small cell lung cancer. J Nucl Med. 2005;46(8):1342-8.

30. Nielsen T, Mouridsen K, Maxwell RJ, Stødkilde-Jørgensen H, Østergaard L, Horsman MR. Segmentation of dynamic contrast enhanced magnetic resonance imaging data. Acta Oncol. 2008;47(7):1265-70.

31. Yamazoe S, Takahara T, Shimizu K, Ouchi K, Mogami T, Harada J, Fukuda K: Diffusion-weighted imaging with relative signal intensity statistical thresholding for delineating prostate cancer tumors. Magn Reson Med Sci 2012, 11(1):1-8.

32. Fan J, Yau DK, Elmagarmid AK, Aref WG. Automatic image segmentation by integrating color-edge extraction and seeded region growing. IEEE Trans Image Process. 2001:10(10):1454-66.

33. Nam H, Huh SJ, SG J, Park W, Lee JE, Choi JY, Kim B-T, Kim CK, Park BK. 18Ffluorodeoxyglucose positron emisson tomography/computed tomography guided conformal brachytherapy for cervical cancer. Int J Radiat Oncol Biol Phys. 2012;84(1):e29-34.

34. Chao A, Ho K-C, Wang C-C, Cheng H-H, Lin G, Yen T-C, Lai C-H. Positron emission tomography in evaluating the feasibility of curative intent in cervical cancer patients with limited distant lymph node metastases. Gynecol Oncol. 2008;1 10(2):172-8.

35. Mundt AJ, Mell LK, Roeske JC. Preliminary analysis of chronic gastrointestinal toxicity in gynecology patients treated with intensity-modulated whole pelvic radiation therapy. Int J Radiat Oncol Biol Phys. 2003;56(5):1354-60

36. Gill BS, Pai SS, McKenzie S, Beriwal S. Utility of PET for radiotherapy treatment planning. PET clinics. 2015;10(4):541-54

37. Zhang S, Xin J, Guo Q, Ma J, Ma Q, Sun H, Zhao X. Defining PET tumor volume in cervical cancer with hybrid PET/MRl: a comparative study. Nucl Med Commun. 2014;35(7):712-9.

38. Olsen JR, Esthappan J, DeWees T, Narra VR, Dehdashti F, Siegel BA, Schwarz JK, Grigsby PW. Tumor volume and subvolume concordance between FDGPET/CT and diffusion-weighted MRI for squamous cell carcinoma of the cervix. J Magn Reson Imaging. 2013;37(2):431-4.

39. Buijsen J, van den Bogaard J, van der Weide H, Engelsman S, van Stiphout R, Janssen M, Beets G, Beets-Tan R, Lambin P, Lammering G. FDG-PET-CT reduces the interobserver variability in rectal tumor delineation. Radiother Oncol. 2012;102(3):371-6.

40. Tchelebi L, Ashamalla H. Overcoming the hurdles of using PET/CT for target volume delineation in curative intent radiotherapy of non-small cell lung cancer. Ann translational Med. 2015;3(14).

41. Van Baardwijk A, Bosmans G, Boersma L, Buijsen J, Wanders S, Hochstenbag M, Van Suylen R-J, Dekker A, Dehing-Oberije C, Houben R. Pet-ct-based auto-contouring in non-small-cell lung cancer correlates with pathology and reduces interobserver variability in the delineation of the primary tumor and involved nodal volumes. Int J Radiat Oncol Biol Phys. 2007;68(3):771-8.

\section{Submit your next manuscript to BioMed Central and we will help you at every step:}

- We accept pre-submission inquiries

- Our selector tool helps you to find the most relevant journal

- We provide round the clock customer support

- Convenient online submission

- Thorough peer review

- Inclusion in PubMed and all major indexing services

- Maximum visibility for your research

Submit your manuscript at www.biomedcentral.com/submit
C Biomed Central 